\title{
UMA ABORDAGEM EDUCACIONAL ACERCA DO PROCESSO DE MODELAGEM FÍSICA-MATEMÁTICA NA ENGENHARIA
}

DOI: 10.37702/2175-957X.COBENGE.2021.3555

Natan Sian das Neves - natan.sian@gmail.com

Universidade Federal do Rio de Janeiro

Rua Santa Rosa 136

29164-302 - serra - ES

Resumo: A área da física-matemática apresenta uma vasta quantidade de modelos teóricos correlacionados aos fenômenos físicos presentes na engenharia. Diversos fenômenos apresentam características particulares e complexidades, que tornam a solução mais sofisticada e exigente. Desta forma, os discentes devem ser capazes de compreender o funcionamento do sistema metodológico do processo de modelagem de engenharia. Este artigo concentra-se na elaboração de um estudo teórico, com base em uma ótica de ensino-aprendizado, acerca do processo de modelagem física-matemática de problemas engenharia. Utiliza-se um caso-teste para exemplificar o processo de solução e as hipóteses simplificadoras utilizadas. Com base nas análises e discussões desenvolvidas ao longo do trabalho pode-se concluir alguns pontos acerca da importância dos aspectos teóricos do processo de modelagem correlacionado ao ensino em cursos de engenharia.

Palavras-chave: modelagem, física-matemática, ensino, engenharia 


\section{UMA ABORDAGEM EDUCACIONAL ACERCA DO PROCESSO DE MODELAGEM FÍSICA-MATEMÁTICA NA ENGENHARIA}

\section{INTRODUÇÃO}

Ao longo da formação acadêmica dos discentes em cursos de engenharia (e.g.: civil, mecânica, elétrica e química), eles são expostos a diferentes áreas de aplicação da engenharia, que apresentam inúmeros problemas físicos de interesse prático e científico, por exemplo, os problemas relacionados ao campo da transferência de calor, mecânica das fluidos e solos, estrutural e dentre outros. Frequentemente, diversos desses problemas de engenharia podem ser governados por equações diferenciais (ordinárias ou parciais), que apresentam naturezas, complexidades particulares e comportamentos não lineares. Em razão dessas características, recorrentemente, a engenharia realiza simplificações nos modelos físicos, visando simplificar a solução do problema (FORTUNA, 2000).

Neste contexto, o conhecimento das hipóteses e considerações adotadas ao longo do processo de modelagem física-matemática de problemas de engenharia é fundamental para a uma formação sólida dos discentes. Em muitas circunstâncias, por conta da forte exigência de uma base conceitual, de comportamento físico e de matemática abstrata, o processo de ensino-aprendizagem de disciplinas que necessitam do desenvolvimento de uma modelagem física-matemática pode sofrer defasagens ao longo do caminho (PINHEIRO et al.; 2017). Desta maneira, este artigo visa apresentam uma discussão prática com enfoque didático sobre o processo sistemático de modelagem física-matemática de problemas de engenharia, visando simplificar o aprendizado dos discentes.

\section{PROCESSO DE MODELAGEM DE PROBLEMAS FÍSICOS E ASPECTOS EDUCACIONAIS NA ENGENHARIA}

O processo de modelagem de um problema de engenharia pode apresentar inúmeras complexidades dependendo do nível de dificuldade do problema estudado. Como este trabalho visa apresentar um panorama geral simplificado, visando demonstrar didaticamente as principais ideias e considerações adotadas ao longo da solução do problema.

Figura 1 - Configurações e dimensões do problema físico de engenharia de interesse.

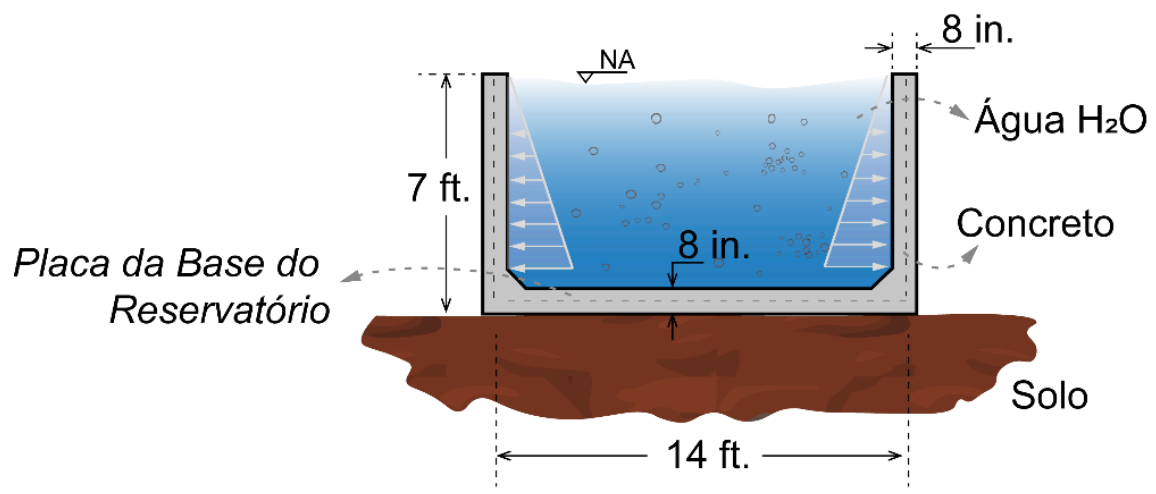

Fonte: Elaborado pelo Autor (2021). 
Neste contexto, buscando amplificar o entendimento da proposta do trabalho, a discussão e análises são feitas com base em um exemplo numérico, onde são apresentadas as ideias gerais. Primeiramente, a modelagem física-matemática de engenharia inicia a partir da definição do problema físico de interesse. O problema proposto consiste em reservatório de água em contato com solo, conforme mostra a Figura 1. Após a definição do problema, realiza-se uma avaliação preliminar acerca das variáveis de interesse. A placa da base (parte inferior da estrutura em contato direto com o solo) pode ser representada como uma viga elástica de fundação. Nesse exemplo, busca-se estimar, na parte inferior da estrutura, o diagrama de momento fletor $M(x)$.

A natureza da estrutura é tridimensional, todavia, em diversos problemas de engenharia, por conta das características físicas e geométricas, pode-se reduzir a dimensão da análise, em outras palavras, realizar uma modelagem em um domínio bidimensional ou unidimensional. No problema proposto, o comportamento estrutural do reservatório é descrito por um modelo reticulado de viga sob base elástica (ou viga de fundação) - com base na teoria elástica de vigas de Euler-Bernoulli, conforme esquematizado na Figura 2. Esse fator é um ponto importante no processo de ensino, uma vez que é o discente deve ser capaz de analisar as dimensões/características relevantes no problema, visando encontrar a configuração adequada para a solução.

Figura 2 - Esquema didático do processo de simplificação de redução da ordem do modelo estrutural.

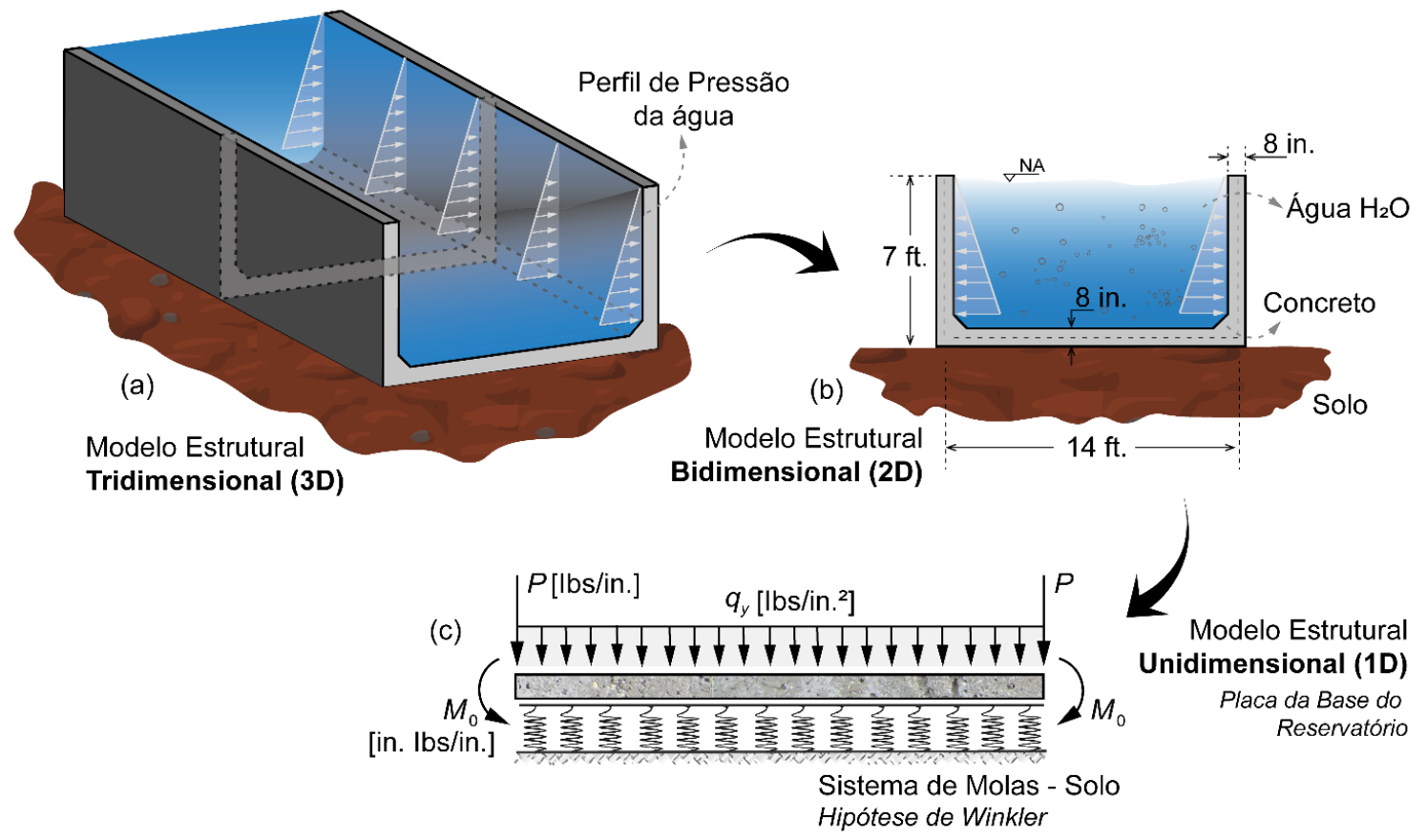

Fonte: Elaborado pelo Autor (2021).

Os passos apresentados até o momento podem ser entendidos como uma avaliação preliminar do problema, onde são analisadas as características, variáveis, dimensões gerais do problema e possíveis modelos de estruturais de análise. Após esse estágio, iniciase o refinamento e construção do modelo, onde são realizados os levamentos de cargas, tipos de solicitações, características geométricas, tipos de materiais empregados e métodos de soluções.

Primeiramente, conforme apresentado na Figura 2, o modelo estrutural adotado para 
solucionar o problema proposto é descrito pela teoria de viga sob base elástica, a equação diferencial que representa o fenômeno físico é mostrada a seguir:

$$
\frac{d^{2}}{d x^{2}}\left[E I \frac{d^{2} w}{d x^{2}}\right]+k_{v} w=q_{y}(x)
$$

onde:

$w=$ deslocamento da viga;

$E=$ módulo de elasticidade do material;

$I=$ momento de inércia da seção transversal;

$k_{v}=$ coeficiente vertical do solo;

$q_{y}=$ carregamento distribuído ao longo da viga.

Outro fator importante que os discentes devem compreender ao longo de um processo de modelagem é o modelo físico que descreve o comportamento material. O solo é descrito pela hipótese de Winkler - que estabelece que a reação do solo é proporcional ao deslocamento, permitindo considerar um sistema de molas lineares independentes. Além disso, na construção da Equação (1), adota-se a lei de Hooke - que estabelece que o material é modelado em regime elástico-linear, garantindo a proporcionalidade entre tensão-deformação (DEN HARTOG, 1987).

Após definir o modelo estrutural e suas características gerais, realiza-se uma catalogação das propriedades dos materiais que compõem o sistema estrutural. No reservatório, adota-se dois materiais, um líquido (água) e um sólido (concreto), conforme esquematizado na Figura 1. O módulo de elasticidade do material sólido, que é utilizado na construção da estrutura do reservatório, é igual a $1512 \mathrm{lbs} / \mathrm{in}^{2}$. A massa específica do material da água e do concreto são, respectivamente, iguais a $0,0361 \mathrm{lbs} / \mathrm{in}^{3}$ e 0,0875 Ibs/in ${ }^{3}$. Além disso, o coeficiente do solo (ou da base elástica) é igual a $165 \mathrm{lbs} / \mathrm{in}^{3}$.

Computando os carregamentos resultantes aplicados na região inferior da estrutura (ver Figura 2), têm-se os seguintes valores:

(i) Peso da placa da base: 8 in $\times 0,0857 \mathrm{lbs} / \mathrm{in}^{3}=0,70 \mathrm{lbs} / \mathrm{in}^{2}$

(ii) Peso da água: 84 in $\times 0,0361 \mathrm{lbs} / \mathrm{in}^{3}=3,04 \mathrm{lbs} / \mathrm{in}^{2}$

(iii) Carga uniformemente distribuída: $0,70+3,04=3,74 \mathrm{lbs} / \mathrm{in}^{2}$

(iv)Peso da parede: 86 in $\times 8$ in $\times 0,0875 \mathrm{lbs} / \mathrm{in}^{3}=60 \mathrm{lbs} / \mathrm{in}$

(v) Momento devido ao perfil de pressão da água na parede:

0,5 in $x 84$ in $x\left(0,0361 \mathrm{lbs} / \mathrm{in}^{3} \times 84\right.$ in $) \times(1 / 3) \times 84$ in $=3570$ in lbs/in

A fase de levamento de cargas é um estágio importante na modelagem de um problema físico de engenharia, devendo ser realizada com cautela, a fim de avaliar corretamente os valores e os tipos de solicitações na estrutura (e.g.: forças e momentos concentrados, cargas distribuídas, carregamentos superficiais, ações térmicas e dentre outros). Vale frisar que conforme exposto anteriormente, como a estrutura original é tridimensional, as unidades dos carregamentos aplicados dependem da profundida do reservatório.

Definido os carregamentos, o tipo de modelo estrutural, as características físicas e geométricas, o passo seguinte na modelagem é a definição do modo de solução do problema. Na engenharia, usualmente, pode-se dividir os métodos de soluções em três classes: soluções analíticas, numéricas e experimentais. 
Primeiramente, os ensaios em laboratórios buscam descrever, em escala real ou reduzida, um problema encontrado na natureza, sendo que esse tipo de procedimento fornece resultados com maior grau de realismo para análise do comportamento do fenômeno físico. Contudo, a ciência experimental enfrenta obstáculos, como o elevado custo financeiro em relação à mão de obra e equipamentos, espaço físico de execução, dificuldades nas medições e problemas de escala.

Os métodos analíticos e numéricos compõem, basicamente, a classe dos métodos teóricos. Um modelo matemático visa representar um fenômeno real da natureza, sendo utilizados princípios conservativos, leis físicas, relações constitutivas, condições de contorno, dentre outros aspectos. Contudo, como os problemas físicos possuem inúmeras complexidades, diversas aplicações necessitam considerar hipóteses de engenharia, acarretando em modelos simplificados. Dependendo das aproximações adotadas, esses modelos podem fornecer resultados aceitáveis para predizer a realidade ou simplificações acerca do comportamento do problema, exigindo um aprimoramento dos parâmetros da construção do modelo. Além disso, é comum encontrar soluções analíticas somente para problemas específicos, com geometrias e processos físicos simplificados, onde são frequentemente usadas para calibragem de um programa computacional.

Figura 3 - Ideia da programação orientada à eventos e Interface gráfica do programa de cálculo desenvolvido.
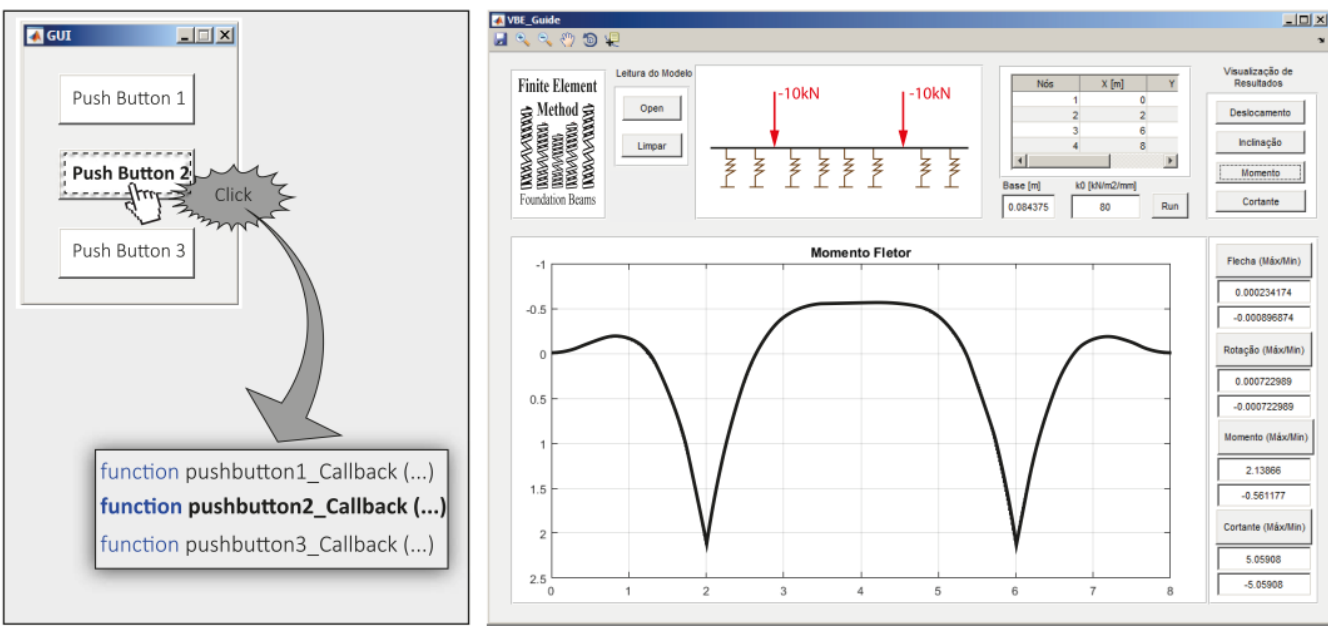

Fonte: Elaborado pelo Autor (2021).

A maioria dos problemas físicos de engenharia apresenta comportamento não trivial, por exemplo, características não lineares, geometrias irregulares, processos transientes, acoplamentos físicos e matemáticos. A dificuldade de encontrar soluções analíticas desses problemas direcionam para soluções aproximadas por meio de técnicas numéricas. Deste modo, o uso de métodos aproximados é uma excelente alternativa a solução de problemas com maior nível de complexidade, uma vez que os computadores estão com sua capacidade em crescente evolução. Neste contexto, dentre as técnicas aproximadas usuais na engenharia, como pioneiro, o método das diferenças finitas (MDF) destaca-se pela sua simplicidade e eficiência na solução. A seara computacional ganhou ferramentas robustas de solução por meio do surgimento, em diferentes períodos, do método dos elementos finitos (MEF) e o método dos volumes finitos (MVF), expandindo a solução de problemas em inúmeras áreas da engenharia, como na mecânica dos sólidos e dos fluidos. Com o avanço matemático e numérico desenvolveu-se otimizações e melhorias, corroborando para os surgimentos de novos métodos, como o método dos elementos de contorno (MEC) e o método meshless. 
Para solução do problema proposto, descrito na equação diferencial de viga de fundação (ver Equação (1)), pode-se aplicar tanto os métodos analíticos como os numéricos. No caso da escolha dos métodos numéricos, pode-se utilizar os procedimentos numéricos de solução com base no método dos elementos finitos. Para a solução da equação diferencial realiza-se uma aproximação do campo de deslocamento por meio dos polinômios cúbicos de Hermite (REDDY, 1993). Em Neves (2019), pode-se encontrar mais informações e detalhamentos matemáticos acerca da formulação numérica de vigas sob base elástica.

Ao nível de ensino de engenharia, é interessante apresentar ao discente um programa computacional com uma interface gráfica educacional, simples e chamativa. Sendo assim, NEVES et al. (2019) apresentam a construção de uma ferramenta para cálculo simples e interativa de vigas de fundação - elaborada em um ambiente que permite ao usuário uma interação direta com o programa via elementos gráficos (botões, slider, menus, etc.). A dinâmica do programa é realizada por eventos, ou seja, as diferentes ações provenientes do usuário sobre elementos gráficos da interface. Cada evento está interligado por uma função denotada Callback, como mostra o esquema da Figura 3.

A interface gráfica do programa de simulação é ilustrada na Figura 3. Os resultados são apresentados ao usuário por meio do campo de deslocamento, rotação, momento fletor e esforço cortante. Com essa ferramenta computacional, os discentes podem realizar análises limítrofes em relação a influência na resposta para diferentes valores do coeficiente de rigidez do base elástica. Além disso, possibilita ao usuário uma investigação acerca dos valores máximos das variáveis de interesse, sendo um recurso importante para determinar os esforços solicitantes de cálculo ou deslocamentos máximos em dimensionamentos de vigas de fundação. Esses tipos de recursos computacionais são importantes para os docentes em sala de aula, uma vez que possibilitam um dinamismo e engajamento dos discentes no entendimento dos problemas estudados.

Figura 4 - Diagrama de momento fletor da viga de fundação representativa da placa da base do reservatório em contato com solo.

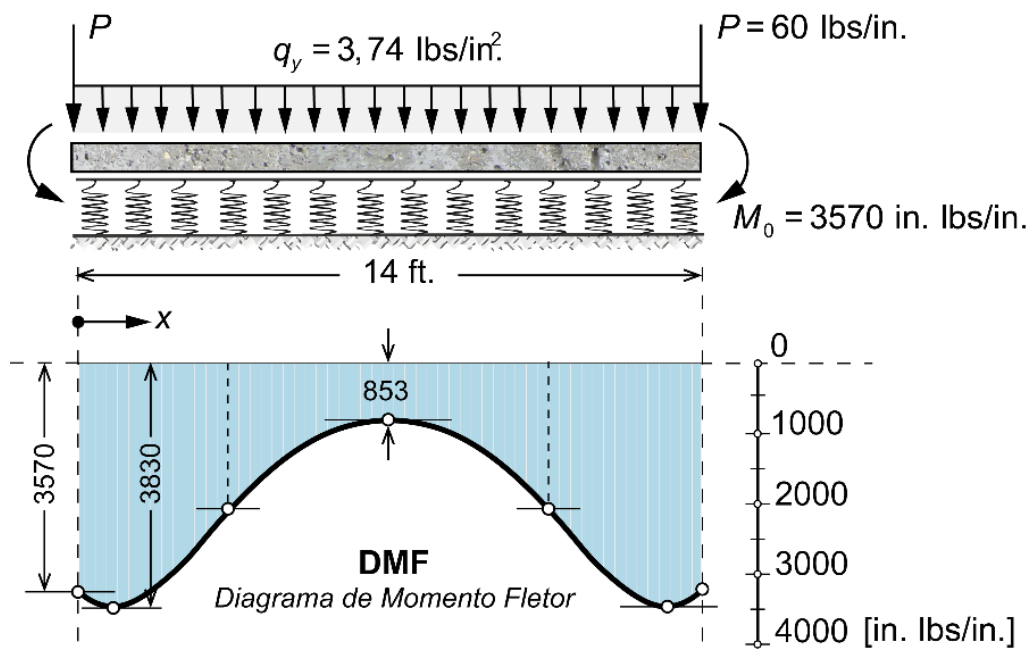

Fonte: Elaborado pelo Autor (2021).

Por outro lado, caso não seja possível solucionar a proposta proposto (ver Figura 1 e 2) com por meio de métodos numéricos. Neste caso estudado em específico, é possível utilizar as soluções analíticas de vigas sob base elástica para cada caso separado das solicitações (carga pontual, momento concentrada e carregamento uniformemente 
distribuído), aplicando o princípio da superposição de efeitos, uma vez que todo o problema é descrito em regime elástico-linear. Uma observação interessante no exemplo estudado é notar que a carga uniformemente distribuída causa somente uma compressão uniforme na fundação (JONES, 1997). Esta sensibilidade física é um fator relevante em um processo de modelagem, possibilitando compreender os efeitos provados pelos carregamentos na estrutura e deve ser apresentado de maneira didática aos discentes.

Desta maneira, para computar os momentos fletores produzidos, respectivamente, pela força $P$ e o momento concentrado $M_{0}$, são apresentados nas expressões matemáticas a seguir:

$$
\begin{gathered}
M^{P}=-\frac{P}{\lambda} \frac{\sinh (\lambda x) \sin \left(\lambda x^{\prime}\right)+\sinh \left(\lambda x^{\prime}\right) \sin (\lambda x)}{\sinh (\lambda l)+\sin (\lambda l)} \\
M^{M_{0}}=\frac{\sinh (\lambda x) \cos \left(\lambda x^{\prime}\right)+\cosh (\lambda x) \sin \left(\lambda x^{\prime}\right)+\sinh \left(\lambda x^{\prime}\right) \cos (\lambda x)+\cosh \left(\lambda x^{\prime}\right) \sin (\lambda x)}{\left(1 / / M_{0}\right) \sinh (\lambda l)+\sin (\lambda l)}
\end{gathered}
$$

onde $\lambda=\sqrt[4]{k / 4 E I}$ e $x^{\prime}=l-x$. O momento resultante é a soma das contribuições dos efeitos provados por $P$ e $M_{0}$, conforme descrito, respectivamente, nas Equações (2) e (3). Desta forma, no exemplo do reservatório, o diagrama de momento fletor da estrutura é apresentado a seguir na Figura 4.

Após obter os resultados requeridos no início do processo de modelagem do problema físico estudado, realiza-se uma verificação dos valores obtidos conforme cada tipo de análise e projeto realizado. Caso os resultados não estejam adequados, deve-se, primeiramente, realizar uma revisão geral de todas as etapas mencionadas anteriormente. $\mathrm{O}$ discente deve ter em mente que a modelagem de um problema de engenharia é um processo sistémico - que deve seguir uma estrutura bem definida. Além disso, vale mencionar também, que em muitas circunstâncias os métodos de solução adotados não são capazes de resolver o problema da maneira adequada, por conta de suas limitações e características especificas, conforme discutido anteriormente no trabalho.

Figura 5 - Fluxograma geral do processo de modelagem física-matemática de engenharia.

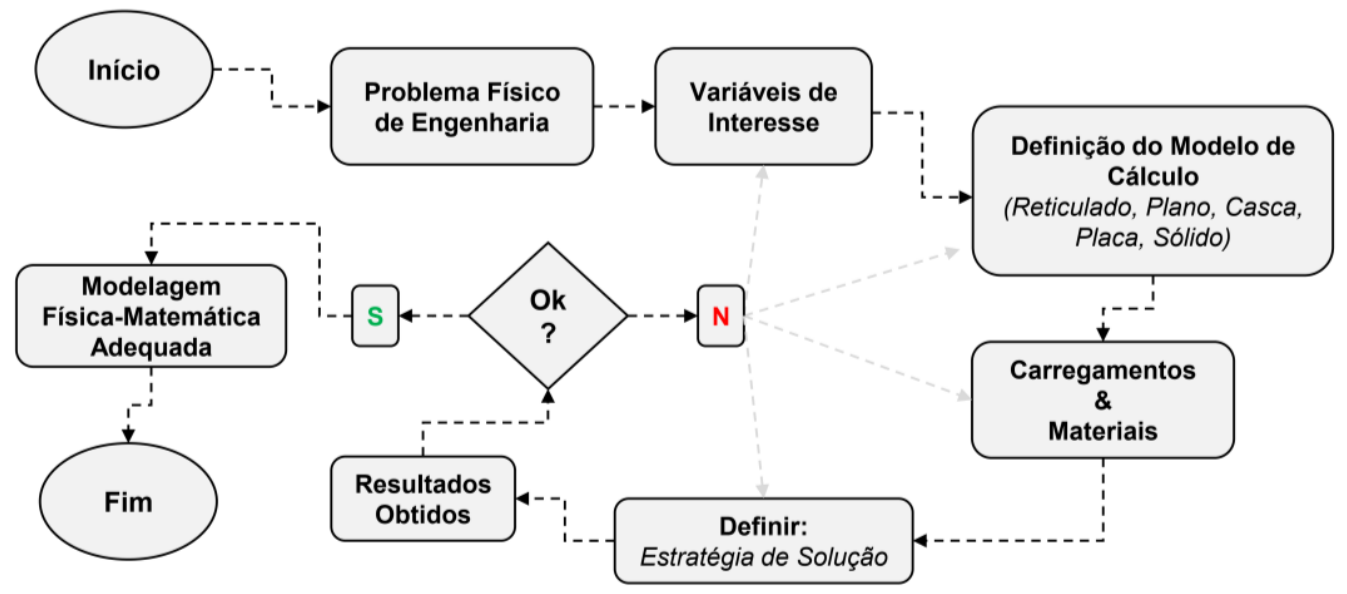

Fonte: Elaborado pelo Autor (2021). 
Para resumir todo o processo de modelagem comentada ao longo do trabalho, apresenta-se, na Figura 5, um fluxograma geral utilizado. Ressalta-se, é claro, que esse fluxograma é um esquema didática e simples de uma modelagem física-matemática, no entanto, ele fornece uma ideia geral para os discentes acerca do tema.

\section{CONSIDERAÇÕES FINAIS}

Este artigo buscou discutir e analisar simplificadamente o processo de modelagem física-matemática de problemas práticos na engenharia, enfatizando os aspectos e impactos no processo de ensino-aprendizado em cursos de engenharia. Com base no exemplo e nas reflexões apontadas ao longo do trabalho, é fundamental que o discente, ao longo de sua trajetória acadêmica, compreenda o funcionamento do processo de modelagem, conhecendo as hipóteses e considerações físicas adotadas no problema, a fim de estabelecer soluções otimizadas e seguras.

Por fim, pode-se concluir que o processo de modelagem de problemas físicos é um pilar fundamental na formação dos alunos de engenharia, fornecendo uma base conceitual, matemática e uma aprimoramento da interpretação comportamental de fenômenos físicos presentes na natureza. Frisando que ainda nos tempos atuais, com disponibilidade de recursos computacionais avançados, o processo de ensino de disciplinas nessa área é complexo, exigindo do docente um conhecimento técnico e acerca das metodologias de ensino.

\section{REFERÊNCIAS}

DEN HARTOG, Jacob Pieter. Advanced strength of materials. Courier Corporation, 1987.

FORTUNA, Armando de Oliveira. Técnicas Computacionais para Dinâmica dos Fluídos Vol. 30. Edusp, 2000.

JONES, Glyn. Analysis of beams on elastic foundations: using finite difference theory. Thomas Telford, 1997.

MATLAB. Version 9.6.0 (R2019a). Natick, Massachusetts: The MathWorks Inc., 2019.

NEVES, Natan Sian. Modelo computacional avançado para análise de estruturas sob ação de gradientes térmicos. Dissertação (Mestrado) - Universidade Federal do Espírito Santo, Vitória, 2019.

NEVES, Natan Sian; PINHEIRO, Vitor Pancieri; CAMARGO, Rodrigo Silveira.

Desenvolvimento de uma interface gráfica educacional para ensino de elementos finitos aplicado a problemas de viga sob base elástica. p. 67-70 . In: X Encontro Científico de Física Aplicada. Anais: São Paulo: Blucher, 2019.

PINHEIRO, Vitor Pancieri; NEVES, Natan Sian; CANDIDO, Daniel Carvalho de Moura; LOEFFLER, Carlos Friedrich. Uma abordagem conceitual da mecânica do contínuo aplicada ao processo de modelagem em física matemática. Revista Ifes Ciência, 3(1), 189-203. 2017. 
REDDY, J. N. An introduction to the finite element method. New York: McGraw-Hill, 2010.

\title{
AN EDUCATIONAL APPROACH ABOUT THE PHYSICAL-MATHEMATICAL MODELING PROCESS IN ENGINEERING
}

\begin{abstract}
The area of mathematical physics presents a vast number of theoretical models correlated to the physical phenomena present in engineering. Several phenomena have particular characteristics and complexities, which make the solution more sophisticated and demanding. In this way, students must be able to understand the operation of the methodological system of the engineering modeling process. This article focuses on the elaboration of a theoretical study, based on a teaching-learning perspective, about the process of physical-mathematical modeling of engineering problems. A test case is used to exemplify the solution process and the simplifying assumptions used. Based on the analyzes and discussions developed throughout the work, it is possible to conclude some points about the importance of the theoretical aspects of the modeling process correlated to teaching in engineering courses.
\end{abstract}

Keywords: modeling, mathematical physics, teaching, engineering. 